\title{
Article
}

\section{Stochastic transients as a source of quasi-periodic processes in the solar atmosphere}

Yuan, Ding, Su, Jiangtao, Jia, Fangran and Walsh, Robert William Available at https://clok.uclan.ac.uk/18044/

Yuan, Ding orcid iconORCID: 0000-0002-9514-6402, Su, Jiangtao, Jia, Fangran and Walsh, Robert William orcid iconORCID: 0000-0002-1025-9863 (2016) Stochastic transients as a source of quasi-periodic processes in the solar atmosphere. The Astrophysical Journal Supplement Series, 224 (2). ISSN 0067-0049

It is advisable to refer to the publisher's version if you intend to cite from the work. http://dx.doi.org/10.3847/0067-0049/224/2/30

For more information about UCLan's research in this area go to http://www.uclan.ac.uk/researchgroups/ and search for <name of research Group>.

For information about Research generally at UCLan please go to http://www.uclan.ac.uk/research/

All outputs in CLoK are protected by Intellectual Property Rights law, including Copyright law. Copyright, IPR and Moral Rights for the works on this site are retained by the individual authors and/or other copyright owners. Terms and conditions for use of this material are defined in the policies page.

\section{CLoK}

Central Lancashire online Knowledge www.clok.uclan.ac.uk

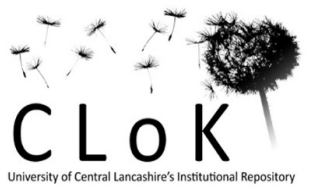




\title{
STOCHASTIC TRANSIENTS AS A SOURCE OF QUASI-PERIODIC PROCESSES IN THE SOLAR ATMOSPHERE
}

\author{
Ding Yuan ${ }^{1,2}$, Jiangtao $\mathrm{Su}^{2}$, FAngran JiaO $^{3}$, And Robert W. Walsh ${ }^{1}$ \\ ${ }^{1}$ Jeremiah Horrocks Institute, University of Central Lancashire, Preston PR1 2HE, UK; DYuan2@uclan.ac.uk \\ ${ }^{2}$ Key Laboratory of Solar Activity, National Astronomical Observatories, Chinese Academy of Sciences, Beijing, 100012, China \\ ${ }^{3}$ Shandong Provincial Key Laboratory of Optical Astronomy and Solar-Terrestrial Environment, Institute of Space Sciences, Shandong University, Weihai, 264209 \\ Shandong, China \\ Received 2016 February 18; accepted 2016 March 31; published 2016 June 8
}

\begin{abstract}
Solar dynamics and turbulence occur at all heights of the solar atmosphere and could be described as stochastic processes. We propose that finite-lifetime transients recurring at a certain place could trigger quasi-periodic processes in the associated structures. In this study, we developed a mathematical model for finite-lifetime and randomly occurring transients, and found that quasi-periodic processes with periods longer than the timescale of the transients, are detectable intrinsically in the form of trains. We simulate their propagation in an empirical solar atmospheric model with chromosphere, transition region, and corona. We found that, due to the filtering effect of the chromospheric cavity, only the resonance period of the acoustic resonator is able to propagate to the upper atmosphere; such a scenario is applicable to slow magnetoacoustic waves in sunspots and active regions. If the thermal structure of the atmosphere is less wild and acoustic resonance does not take place, the long-period oscillations could propagate to the upper atmosphere. Such a case would be more likely to occur in polar plumes.
\end{abstract}

Key words: magnetohydrodynamics (MHD) - Sun: atmosphere - Sun: corona - Sun: oscillations - waves

\section{INTRODUCTION}

A variety of solar transients and turbulence occur in the layers close to the visible surface of the Sun: convections (Nordlund et al. 2009; Stein 2012), granulations (Rieutord \& Rincon 2010), magnetic reconnections (heatings or flares, Hannah et al. 2011; Cargill et al. 2015), spicular activities (De Pontieu et al. 2007b), etc. These activities have finite lifetimes and repeat at the same location without nominal periodicities. However, the nature of intermittency in conjunction with finite lifetimes could well lead to a quasi-periodic process in the associated structures.

In this paper, we aim to propose the idea that stochastic, finite-lifetime transients could generate quasi-periodic processes in the upper atmosphere of the Sun. This is inspired by the recent discoveries of low-amplitude intermittent transverse oscillations of coronal loops (Anfinogentov et al. 2013, 2015; Nisticò et al. 2013), and the connectivity between spicular activities and quasi-periodic propagating disturbances in coronal holes (Jiao et al. 2015; Samanta et al. 2015). Quasiperiodic fast wave trains may also be launched by intermittent impulsive pertubations to reconnection sites (Liu et al. 2011, 2012; Shen \& Liu 2012; Pascoe et al. 2013; Shen et al. 2013; Yuan et al. 2013; Yang et al. 2015). We demonstrate the feasibility of this idea by studying how stochastic spicules are connected with propagating disturbances observed in coronal holes and active regions (ARs). However, we believe this idea is applicable to other temporal and spatial scales in solar physics and in astrophysics.

Spicules are rapidly evolving elongated transients observed off the solar limb (Beckers 1968; Sterling 2000), while mottles and dynamic fibrils, observed at quiet-Sun and AR plages, respectively, are suggested to be the on-disk counterparts of spicules (Hansteen et al. 2006; De Pontieu et al. 2007a; Rouppe van der Voort et al. 2009). Two classes of spicules are identified by De Pontieu et al. (2007b), although skepticism remains (Sterling et al. 2010; Zhang et al. 2012). Type I spicules are relatively slowly evolving features, and will eventually fall back due to gravity on a timescale of 3-7 minutes (De Pontieu et al. 2007b); Type II spicules will fade into the background within a lifetime of about $45 \mathrm{~s}$ (and a spread of about 10-150 s) (De Pontieu et al. 2007b).

Spicular activity or its on-disk counterpart is believed to be associated with the excessive blueshifted spectral line emission (upward flows) observed at the footpoints of ARs (Del Zanna 2008; Doschek et al. 2008; Hara et al. 2008; Tian et al. 2011). Upon observing persistent upflows and correlated variations in linewidth and intensity, McIntosh \& De Pontieu (2009), De Pontieu \& McIntosh (2010), and Tian et al. (2011) intepreted propagating disturbances observed at footpoints of AR loops as quasi-periodic upflows. However, periodic flows rarely occur in nature, unless they are modulated or waveguided by other thermal or magnetic structures. This idea will be demonstrated in this study. A rival theory involves a propagating slow mode of magnetohydrodynamics (MHD) wave: the propagating disturbances have good periodicity (Nakariakov et al. 2000; King et al. 2003; De Moortel 2009) and a natural source of sunspot oscillations (Tian et al. 2014; Yuan et al. 2014a, 2014b; Su et al. 2016a, 2016b); the disturbances in intensity (or density) and velocity oscillate in phase (Wang et al. 2009a, 2009b); the phase speed is dependent on temperature and always smaller than the local acoustic speed (Marsh et al. 2009; Kiddie et al. 2012; Uritsky et al. 2013); moreover, a propagating slow wave could also produce blueshifted line emission (Verwichte et al. 2010), albeit with half the wave period.

Recent simulations suggest that upflows will inevitably excite slow waves in AR loops and that a blend of both upflows and a slow wave may contribute to the observational features in coronal loops (Ofman et al. 2012; Wang et al. 2013). Fang et al. (2015) demonstrates that impulsive heating at the footpoint of a coronal loop will trigger pressure imbalance and excite high-speed upflows. However, as long as the flow 

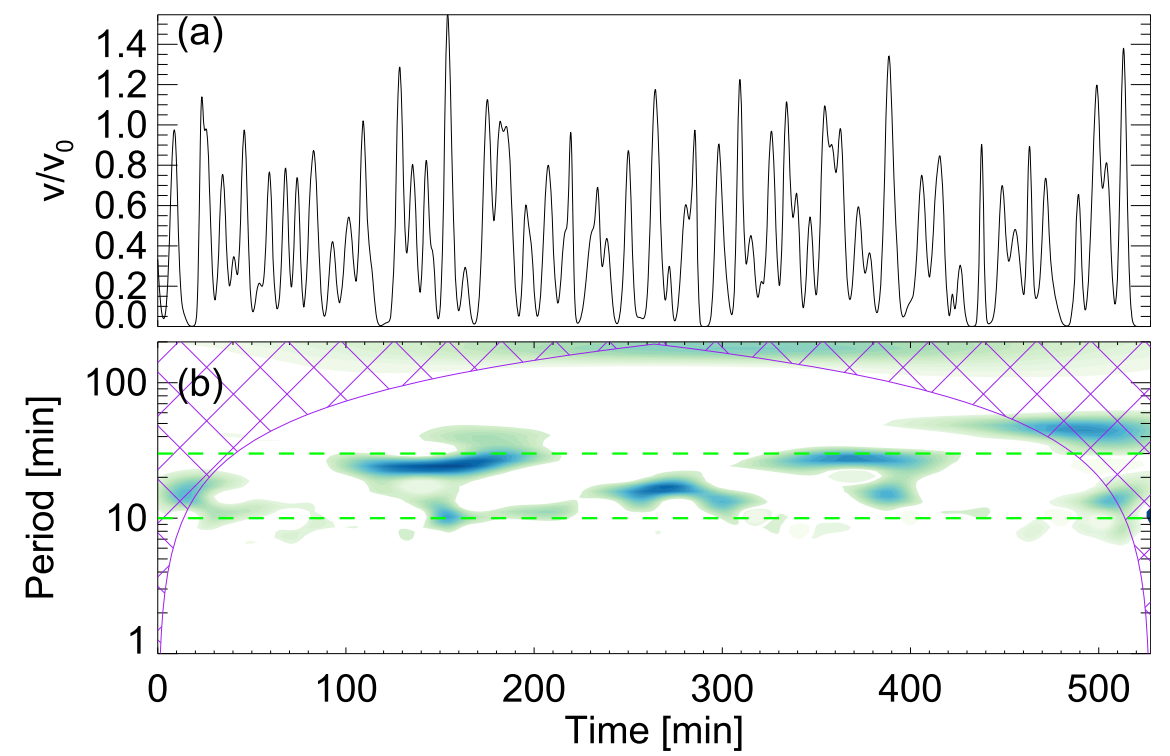

Figure 1. (a) Time series of the stochastic finite-lifetime transient model. (b) The wavelet spectrum illustrates quasi-periodic oscillations in the range of 10-30 minutes, enclosed within the green dashed lines. The cone-of-influence is cross-hatched; within it the spectrum should be considered unreliable. We used $P_{0}=300 \mathrm{~s}$ and $\sigma_{0}=100 \mathrm{~s}$.
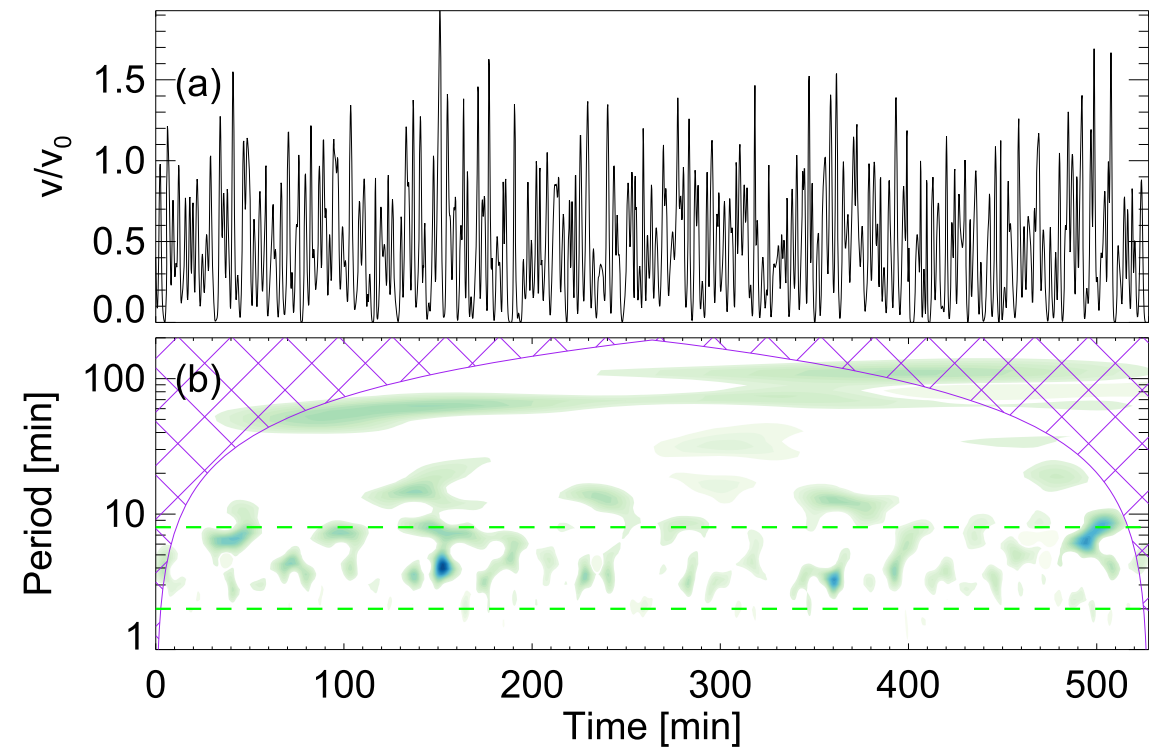

Figure 2. Same as Figure 1, but $P_{0}=80 \mathrm{~s}$ and $\sigma_{0}=30 \mathrm{~s}$. Green dashed lines label the prominent quasi-periodic oscillations in the range of 2-8 minutes. The cone-ofinfluence is cross-hatched; within it the spectrum should be considered unreliable.

become detached from the source, it evolves as a slow wave pulse. Jiao et al. (2015) and Samanta et al. (2015) found compelling evidence that propagating disturbances in polar plumes have strong correlation with the spicular activities at chromospheric height. Similar dynamics (dynamic fibrils) are observed at ARs (Skogsrud et al. 2016).

In this paper, we propose the idea that quasi-periodic processes are an intrinsic part of stochastic finite-lifetime transients, and simulate the propagation of stochastic spicules in the solar atmosphere. Section 2 describes the mathematical model of stochastic transients; Section 3 presents the numerical experiment to demonstrate the idea; Section 4 compares the synthetic data and observations from the Solar Dynamics Observatory $(S D O) /$ Atmospheric Imaging Assembly (AIA) at ARs and polar plumes.

\section{MODEL OF STOCHASTIC TRANSIENTS}

We model a set of sequential transients $g_{i}\left(t_{i}\right)$ with a peak strength at $t_{i}$. For simplicity, we assume that each transient evolves in a Gaussian profile with an amplitude $v_{i}$ and a width $\sigma_{i}$ :

$$
\begin{aligned}
v(t)=\sum_{i=1}^{N} g_{i}\left(t_{i}\right) & =\sum_{i=1}^{N} v_{i} \exp \left[\frac{-\left(t-t_{i}\right)^{2}}{2 \sigma_{i}^{2}}\right], \\
t_{i} & =i P_{0}+\delta t_{i}, \\
\sigma_{i} & =\sigma_{0}+\delta \sigma_{i} .
\end{aligned}
$$

The amplitude $v_{i}$ is a random number that follows a uniform distribution $v_{0} \mathcal{U}(0,1)$. A transient is assumed to be launched every $P_{0}$ and shifted by a normally distributed random offset 


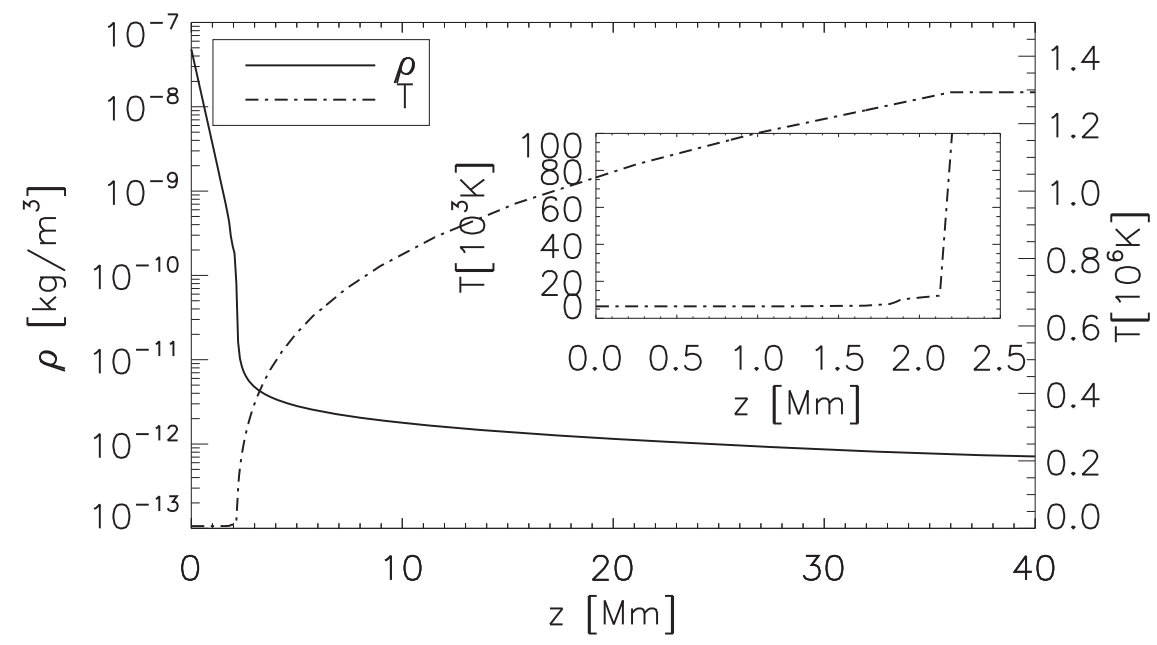

Figure 3. Empirical model of the atmosphere used in this study; the inset enlarges the temperature profile at $z<2.5 \mathrm{Mm}$.

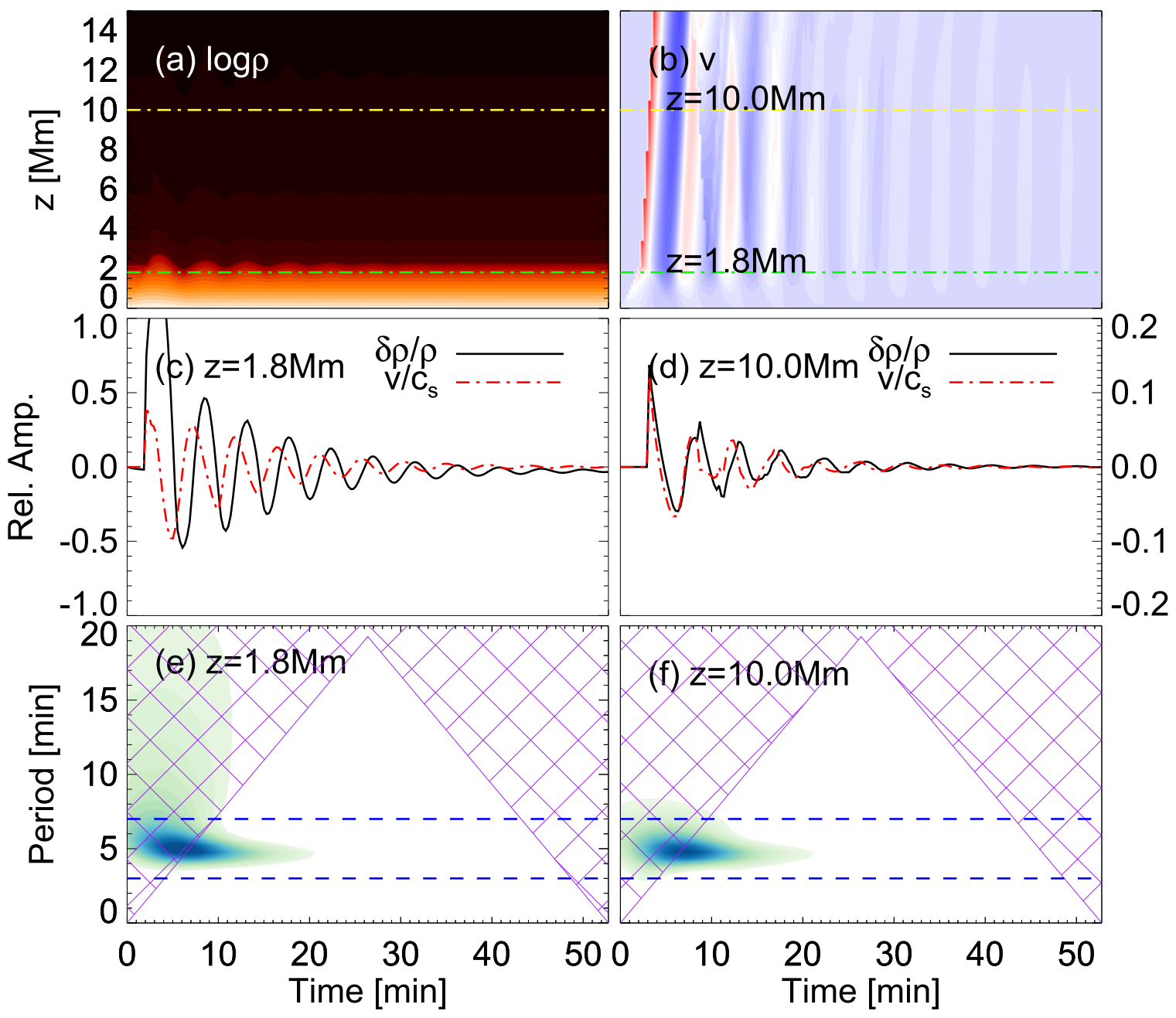

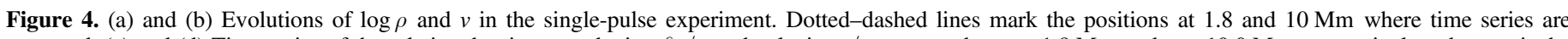

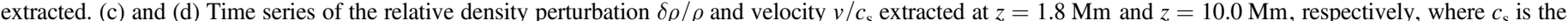

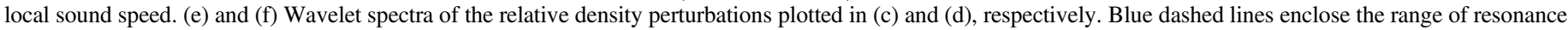
period between 3 and 7 minutes. The cone-of-influence is cross-hatched; within it the spectrum should be considered unreliable.

$\delta t_{i}$, where $\delta t_{i} / P_{1}$ has a normal distribution $\mathcal{N}(0,1)$. So the occurrence interval $t_{i+1}-t_{i}$ has an average value of $P_{0}$ and a standard deviation of $\sqrt{2} P_{1}$. The width $\sigma_{i}$ of a transient, relating to its lifetime, has a nominal value of $\sigma_{0}$ and a random variation $\delta \sigma_{i}$; and $\delta \sigma_{i} / \sigma_{1}$ follows a normal distribution $\mathcal{N}(0,1)$. To reduce the number of free parameters, we fix $P_{1}=P_{0} / 2$, which 


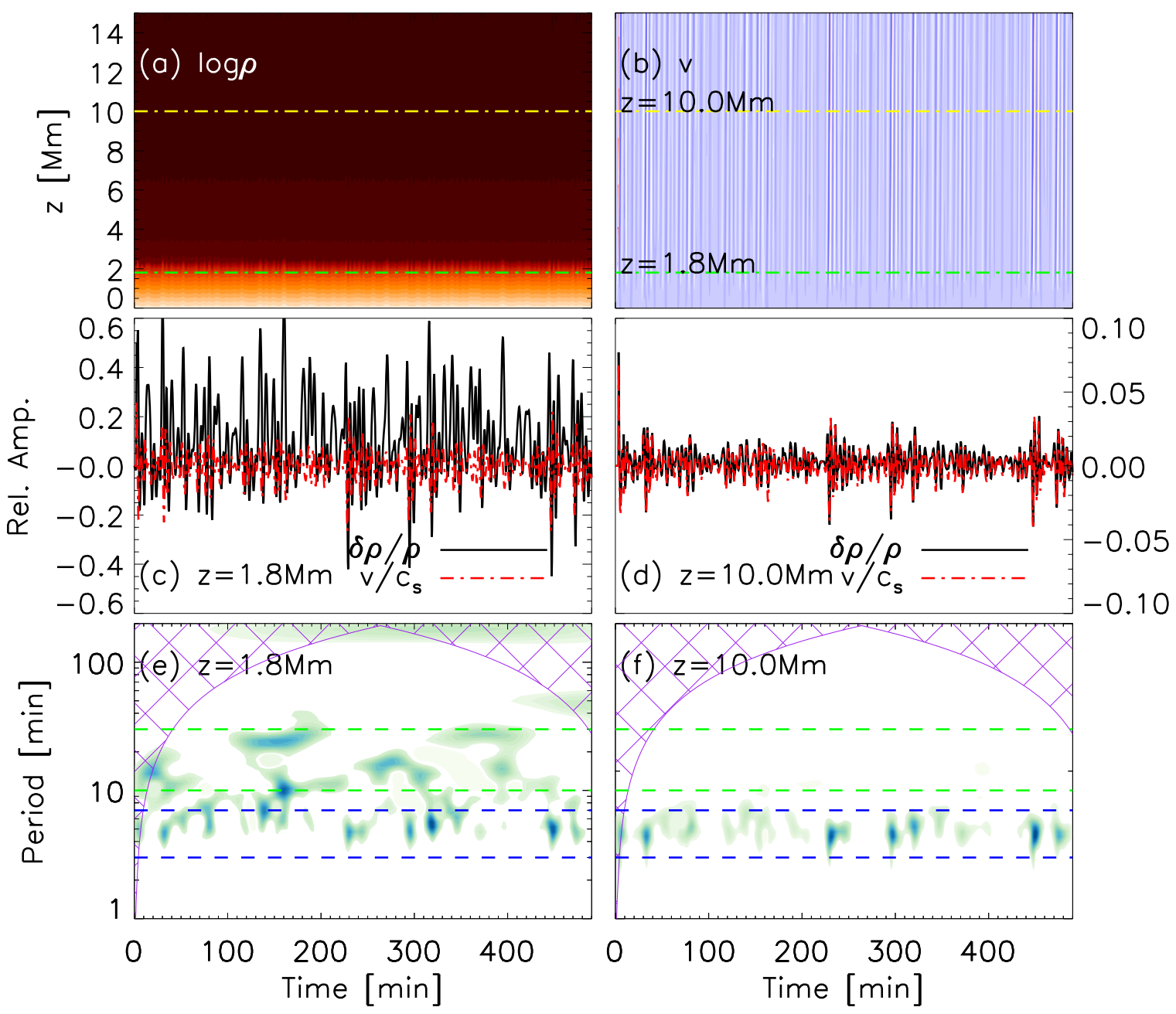

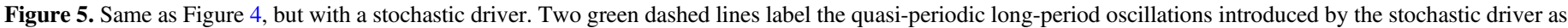

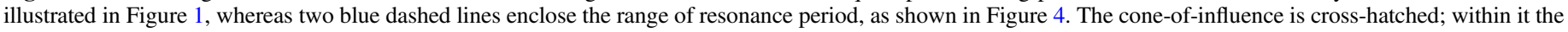
spectrum should be considered unreliable.

allows a small probability that two consecutive transients overlap to some extent. We also set $\sigma_{1}=\sigma_{0} / 4$, so that $\sigma_{i}$ is unlikely to be negative. Tests show that even if $\sigma_{1}$ is set to zero or other reasonable values, the spectrum of the time series remains slightly altered. Similar tests were performed on $P_{1}$ and we did not find significant changes to the spectrum. To see the feasibility of this idea, we introduce typical timescales of the dynamics commonly observed in the lower atmosphere of the Sun.

Type I spicules have dynamic timescales of 3-7 minutes and lifetimes of 2-3 minutes (De Pontieu et al. 2007b); mottles and fibrils are found to have similar timescales (Hansteen et al. 2006; De Pontieu et al. 2007a; Rouppe van der Voort et al. 2009). So we used $P_{0}=300 \mathrm{~s}$ and $\sigma_{0}=100 \mathrm{~s}$ to simulate the time series associated with spicular dynamics, see Figure 1(a). The time series is analyzed by a wavelet transform to show the dynamic spectrum. We applied the Morlet mother function, which is optimized for revealing the oscillatory signals (Torrence \& Compo 1998). In the wavelet spectrum, we plot only the spectral component above the $90 \%$ confidence level. This standard is followed in other wavelet spectra throughout this paper. Spectral peaks aggregate at about $0.5-1.5 \mathrm{mHz}(10-30$ minutes, Figure 1(b)). The power spectrum of quasi-periodic propagating disturbances observed at coronal holes exhibits periodicities in exactly the same frequency range (McIntosh et al. 2010; Banerjee et al. 2011; Krishna Prasad et al. 2011, 2012).

Type II spicules recur more frequently (about every $60 \mathrm{~s}$ ) and are shorter-lived (about $40 \mathrm{~s}$ ). Shorter timescales are found in nanoflares and high-frequency heatings (Porter et al. 1984; Parker 1988; Klimchuk 2015). We use $P_{0}=80 \mathrm{~s}$ and $\sigma_{0}=30 \mathrm{~s}$ to model the dynamics of these timescales; prominent periodicities are found in bands at 2-8 minutes and 50-70 minutes (Figure 2). Short-period propagating disturbances in coronal holes and ARs (e.g., De Moortel 2009; Banerjee et al. 2011; Yuan \& Nakariakov 2012) and sunspot oscillations (e.g., Tian et al. 2014; Yuan et al. 2014a, 2014b; Su et al. 2016a) have periods situated in the range $2-8$ minutes.

\section{NUMERICAL EXPERIMENTS}

In order to explore how stochastic transients affect the upper atmosphere, we create a set of numerical experiments and investigate how the power spectrum is altered observationally throughout its propagation in a stratified atmosphere. We do not attempt to model realistic transients at photospheric temperature and density, but only demonstrate what response could be observed if this sort of perturbation was triggered in the lower atmosphere. 


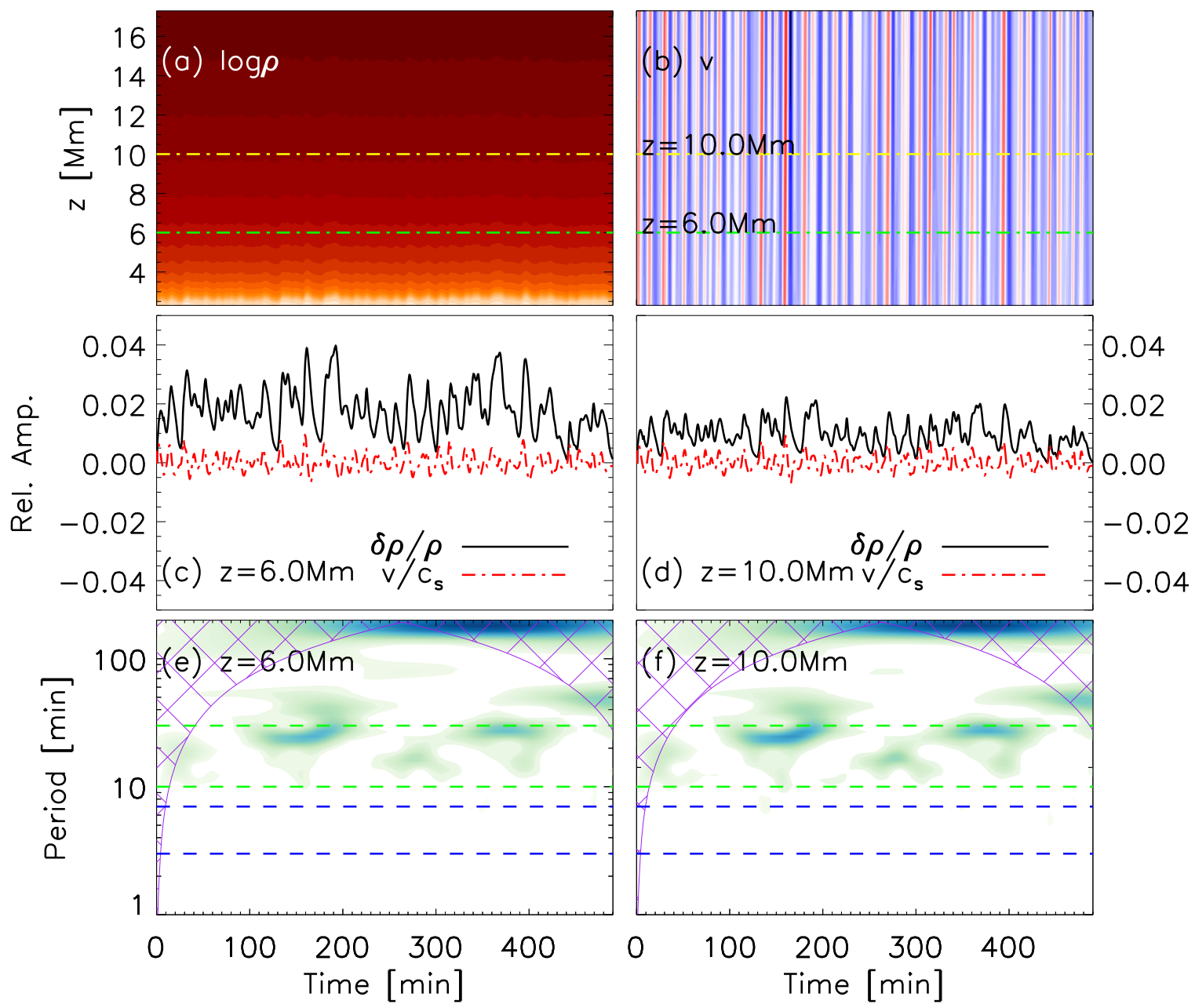

Figure 6. Same as Figure 5, but with the driver implemented at $z=2.3 \mathrm{Mm}$ and time series extracted at $z=6 \mathrm{Mm}$ and $z=10 \mathrm{Mm}$. The green dashed lines label the quasi-periodic long-period oscillations introduced by the stochastic driver as illustrated in Figure 1, whereas the two blue dashed lines enclose the range of resonance period, as shown in Figure 4. The cone-of-influence is cross-hatched; within it the spectrum should be considered as unreliable.

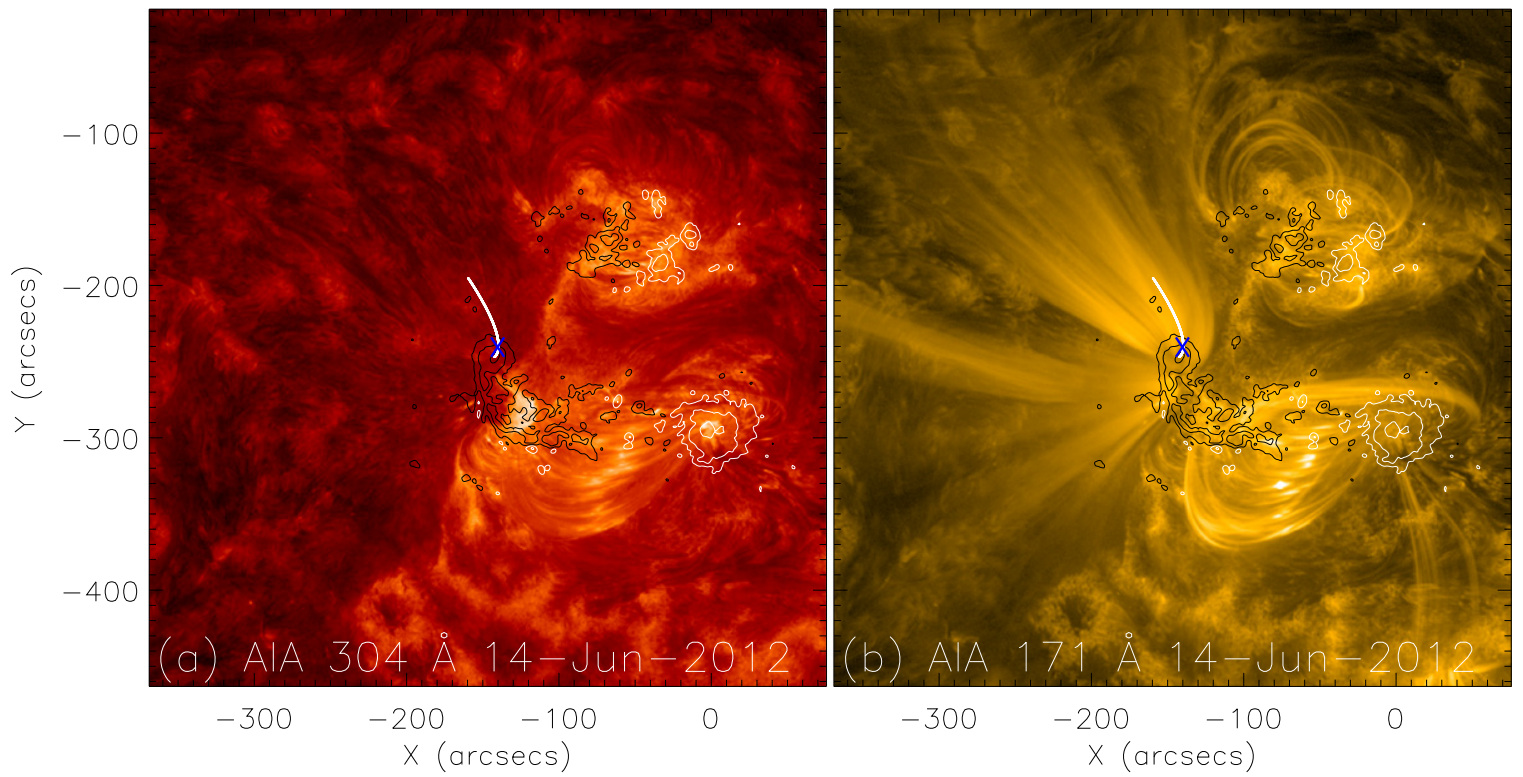

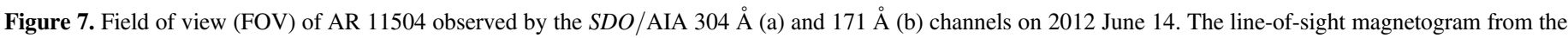

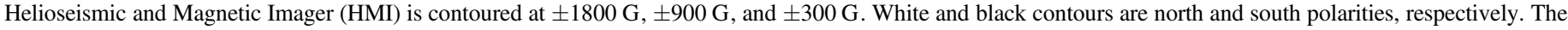
white slice follows a coronal loop, and the blue cross labels the positions where time series are extracted for wavelet analysis in Figure 8. 


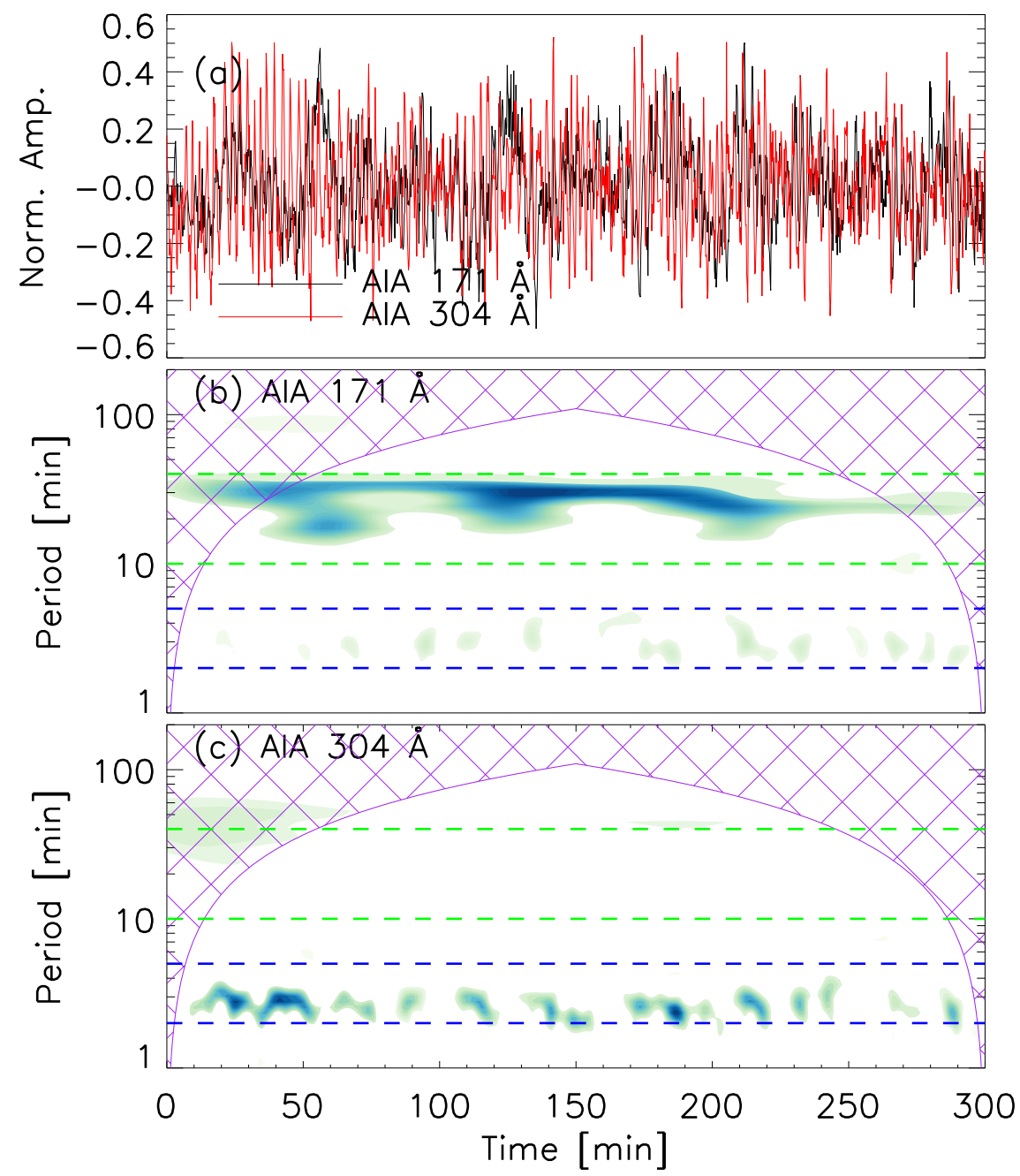

Figure 8. (a) Time series of relative emission intensity extracted at a coronal loop marked in Figure 7. The peak-to-peak amplitude is normalized to \pm 0.5 . (b) and (c) are the wavelet spectra for the AIA $171 \AA$ and $304 \AA$, respectively. The start time is 16:00 UT on 2012 June 14. Two green dashed lines label the quasi-periodic longperiod oscillations at 10-30 minutes, whereas two blue dashed lines enclose oscillations in the band at 3 minutes. The cone-of-influence is cross-hatched; within it the spectrum should be considered unreliable.

\subsection{Empirical Model of the Atmosphere}

Figure 3 plots the one-dimensional model of the atmosphere used in this study. Below $z=2.22 \mathrm{Mm}$, we implemented the mid-age sunspot umbra model of Maltby et al. (1986); close to the bottom boundary $(z<1.2 \mathrm{Mm})$, we set a constant temperature $(6471 \mathrm{~K})$. Above $z=2.22 \mathrm{Mm}$, we adapted the C7 model of Avrett \& Loeser (2008); near the upper boundary $(z \in[36 \mathrm{Mm}, 40 \mathrm{Mm}])$, we set a constant temperature of $1.3 \mathrm{MK}$. This kind of model of temperature has been used in a number of previous studies, e.g., Heggland et al. (2007), Botha et al. (2011), and Snow et al. (2015).

In hydrostatic equilibrium, the pressure is obtained by integrating the gravitational force $\rho g$,

$$
\begin{gathered}
p(z)=p(0) \exp \left[\int_{0}^{z}-\rho g d z\right] \\
p(z)=\frac{\rho(z) k_{\mathrm{B}} T(z)}{0.5 m_{\mathrm{p}}},
\end{gathered}
$$

where $p, \rho$, and $T$ are the plasma pressure, density, and temperature, respectively, $g=275.43 \mathrm{~m} \mathrm{~s}^{-2}$ is the gravitational acceleration at the surface of the Sun, $k_{\mathrm{B}}$ is the
Boltzmann constant, and $m_{\mathrm{p}}$ is the mass of a proton. We choose a base density of $\rho(0)=6.6 \times 10^{-8} \mathrm{~kg} \mathrm{~m}^{-3}$ at $z=0 \mathrm{Mm}$, so at coronal height the density is of the order of $10^{-13} \mathrm{~kg} \mathrm{~m}^{-3}$, see Figure 3. In one-dimensional MHD simulation, the magnetic field decouples from hydrodynamic equilibrium; we used $B=10 \mathrm{G}$, in line with the $z$-axis, in our simulations.

The plasma parameters were normalized by choosing a set of three to be constant: the length $L_{0}=1.0 \mathrm{Mm}$, the density $\rho_{0}=8.0 \times 10^{-10} \mathrm{~kg} \mathrm{~m}^{-3}$, and the magnetic field strength $B_{0}=10 \mathrm{G}$. Then the normalization factor for the velocity is $V_{0}=31.6 \mathrm{~km} \mathrm{~s}^{-1}$, the time $t_{0}=31.7 \mathrm{~s}$, the gravitational constant $g_{0}=997 \mathrm{~m} \mathrm{~s}^{-2}$, and the temperature $T_{0}=0.06 \mathrm{MK}$. So the variables become dimensionless by using $z=\hat{z} L_{0}$, $\rho=\hat{\rho} \rho_{0}, \quad B=\hat{B} B_{0}, \quad t=\hat{t} t_{0}, \quad v=\hat{v} V_{0}, \quad g=\hat{g} g_{0}, \quad p=\hat{p} p_{0}$, $T=\hat{T} T_{0}$ etc., where symbols with a hat are dimensionless variables.

\subsection{Magnetohydrodynamic Simulation}

We used the AMRVAC package (Keppens et al. 2012; Porth et al. 2014) to solve the ideal MHD equations with the finite- 


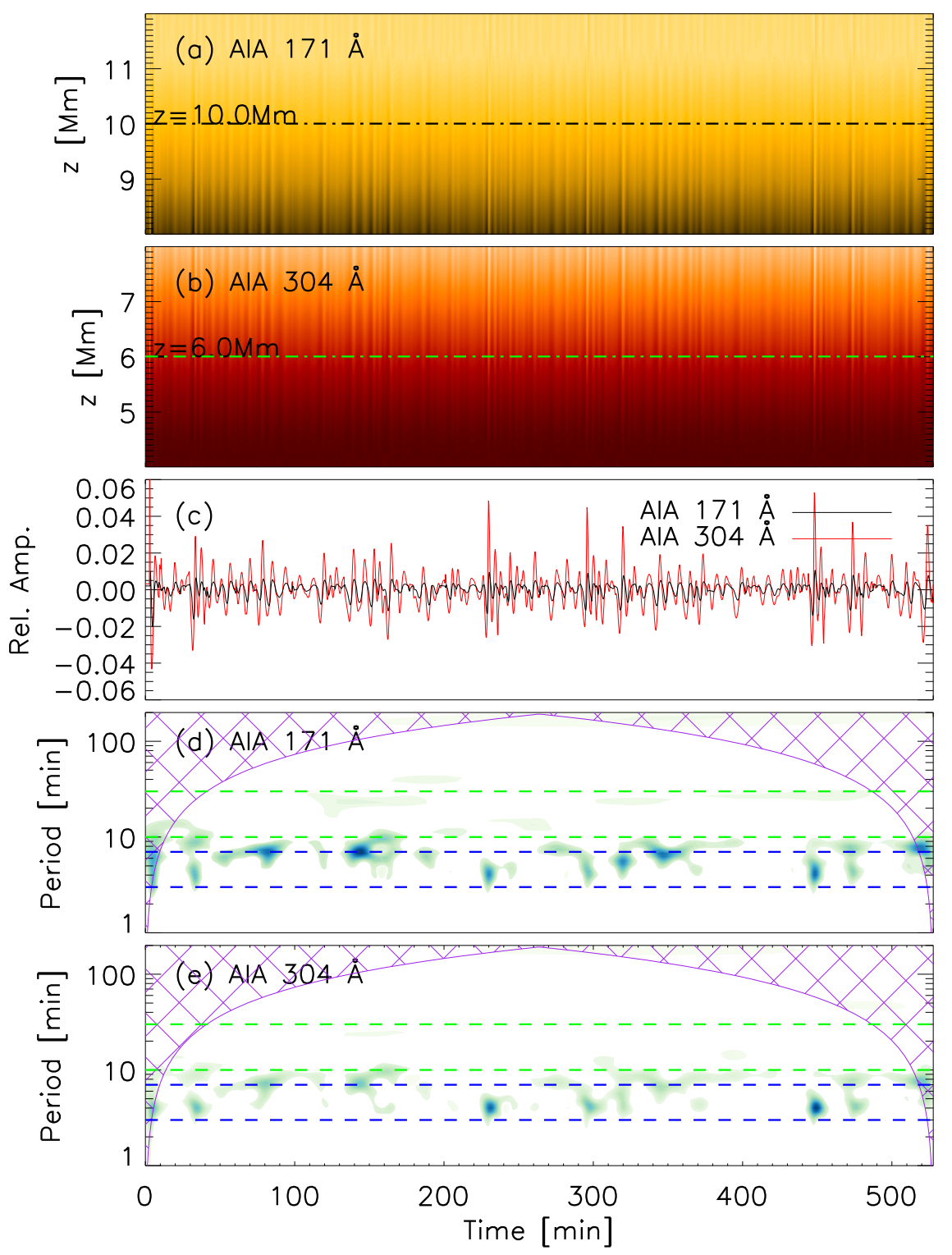

Figure 9. (a) and (b) Evolution of the synthetic AIA $171 \AA$ and $304 \AA$ emission intensities for the simulation with a driver applied at $z=0$ Mm. The black dotteddashed line labels the height at $z=10 \mathrm{Mm}$, where a time series is extracted and analyzed by wavelet transform; the green dotted-dashed line denotes the height at $z=6 \mathrm{Mm}$ where the $304 \AA$ counterpart is obtained: time series and wavelet power spectrum. (c) Relative intensity variations of $304 \AA$ extracted at $z=6 \mathrm{Mm}$ and $171 \AA$ extracted at $z=10 \mathrm{Mm}$. (d) and (e) Wavelet power spectra for the relative intensity variations of $304 \AA$ and $171 \AA$, respectively. The cone-of-influence is crosshatched; within it the spectrum should be considered as unreliable. The green dashed lines label the quasi-periodic long-period oscillations introduced by the stochastic driver as illustrated in Figure 1, whereas the two blue dashed lines enclose the range of resonance period, as shown in Figure 4.

volume method:

$$
\begin{gathered}
\frac{\partial \rho}{\partial t}+\nabla \cdot(\rho \boldsymbol{v})=0, \\
\frac{\partial \rho \boldsymbol{v}}{\partial t}+\nabla \cdot\left[\rho \boldsymbol{v} \boldsymbol{v}+\boldsymbol{I} p_{\text {tot }}-\frac{\boldsymbol{B} \boldsymbol{B}}{\mu_{0}}\right]=\rho \boldsymbol{g}, \\
\frac{\partial \epsilon}{\partial t}+\nabla \cdot\left[\boldsymbol{v}\left(\epsilon+p_{\mathrm{tot}}\right)-\frac{(\boldsymbol{v} \cdot \boldsymbol{B}) \boldsymbol{B}}{\mu_{0}}\right]=\rho \boldsymbol{g} \cdot \boldsymbol{v}, \\
\frac{\partial \boldsymbol{B}}{\partial t}+\nabla \cdot(\boldsymbol{v} \boldsymbol{B}-\boldsymbol{B v})=0, \\
\epsilon=\frac{1}{2} \rho v^{2}+\frac{p}{\gamma-1}+\frac{B^{2}}{2 \mu_{0}},
\end{gathered}
$$

where $\boldsymbol{v}$ is the fluid velocity, $\boldsymbol{B}$ the magnetic field vector, $\boldsymbol{g}=$ $-g z$ the solar gravity vector, $\boldsymbol{I}$ the unit tensor, $\epsilon$ the total energy density, $p_{\text {tot }}=p+B^{2} / 2 \mu_{0}$ the total pressure, $\gamma$ the adiabatic index, and $\mu_{0}$ the magnetic permeability of free space.

The AMRVAC code was configured to solve Equations (6)(10) in Cartesian coordinates $[x, y, z]$. The physical quantities were assumed to be invariant along the $x$ - and $y$-axes (i.e., $\partial / \partial x=\partial / \partial y=0)$. We exploited the HLL approximate Riemann solver (Harten et al. 1983) and implemented the KOREN flux limiter (Koren 1993). A three-step Runge-Kutta method was used in time discretization. This configuration has been used in Yuan et al. (2015a) to study propagating fast waves in randomly structured plasmas.

A solution domain was set at $z \in[0 \mathrm{Mm}, 40 \mathrm{Mm}]$. At the top boundary, we extrapolated values for the density and 


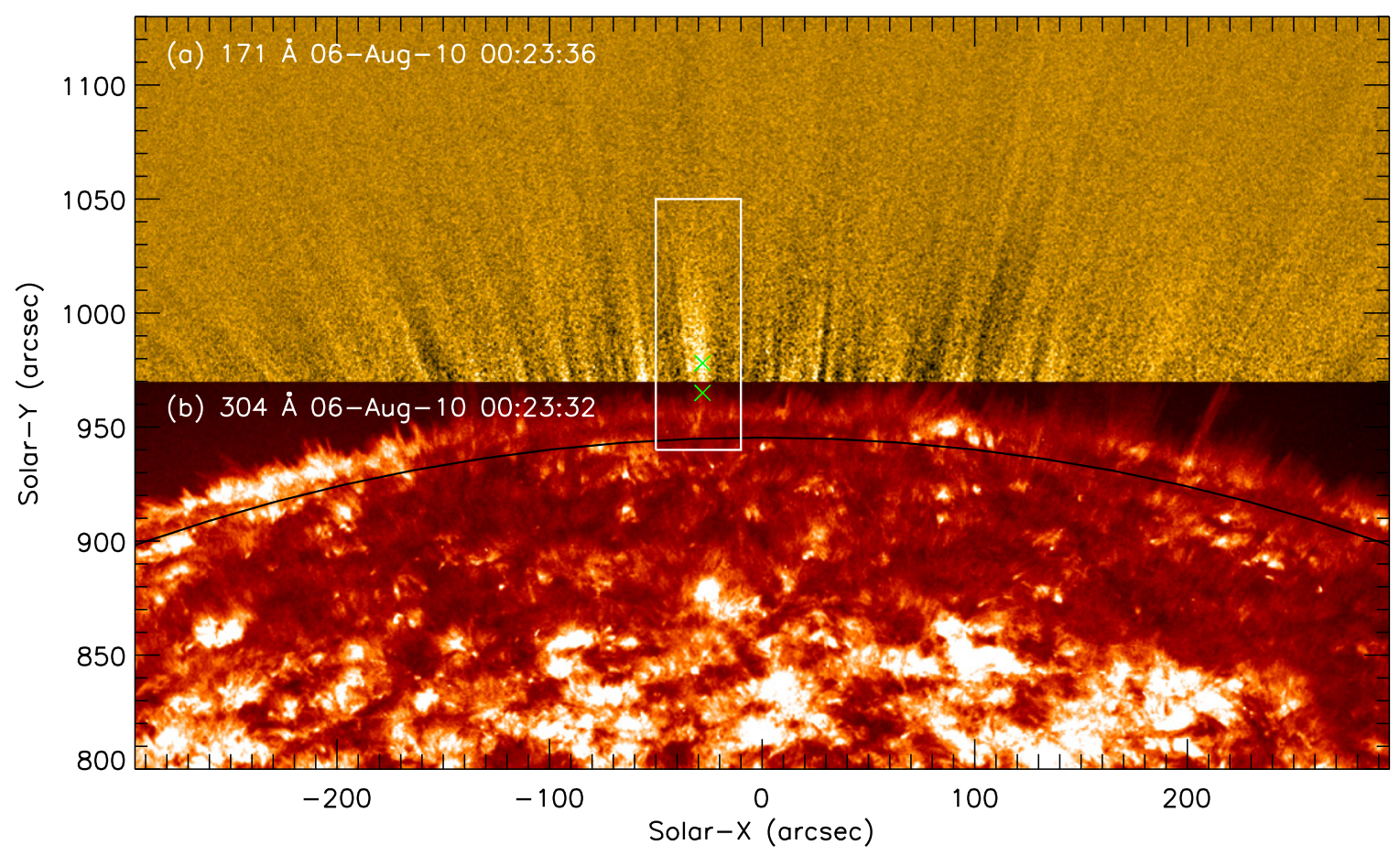

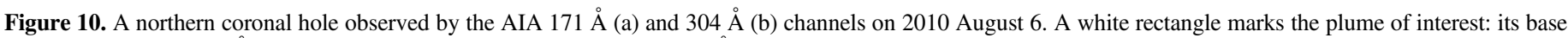

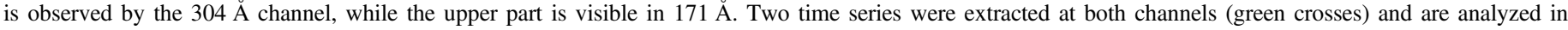
Figure 11 .

pressure; a mirroring boundary condition was used for the velocity to minimize reflections. At the bottom boundary we fixed the values of plasma density and pressure, extrapolated from the empirical model of the atmosphere; the velocity was set up according to the driver model, see Section 2. We used 4000 fixed grid cells without adaptive mesh refinement, so the cell size was $d z=10 \mathrm{~km}$. We relaxed the system until $t /$ $t_{0}=50$; the maximum velocity caused by numerical noise is 0.003 (i.e., $0.1 \mathrm{~km} \mathrm{~s}^{-1}$ ). So we could consider the system to be already in hydrostatic equilibrium, and drivers could be applied at $t / t_{0}=0$.

\subsection{Single-pulse Test}

Our model contains an acoustic resonator for slow magnetoacoustic waves: the slow waves get reflected at the sharp density gradient when propagating downward, and encounter a cut-off effect at the upper atmosphere (Roberts 2006; Afanasyev \& Nakariakov 2015). The theory of the acoustic resonator model can be found in Zhugzhda (2008) and Zhugzhda et al. (1983), and simulations in Botha et al. (2011) and Snow et al. (2015).

A single Gaussian pulse with $\sigma_{i}=100 \mathrm{~s}$ was launched at the bottom boundary. The pulse reached a height of about $2 \mathrm{Mm}$ after about 2 minutes, and the average phase speed was about $15 \mathrm{~km} \mathrm{~s}^{-1}$ (Figure 4(a)). Above $2 \mathrm{Mm}$, the density drops dramatically, and a shock wave started to form, which is the suggested formation mechanism for chromospheric jets and spicules (De Pontieu et al. 2007b; Heggland et al. 2007). The pulse energy did not completely penetrate through the transition region and declined; a significant portion of energy was trapped within the acoustic resonator, bouncing back and forth, see Figure 4(c). The wavelet spectrum of the density perturbation within the resonator $z=1.8 \mathrm{Mm}(T \sim 7000 \mathrm{~K}$, Figure 4(e)) reveals that the pulse initially carried signals over a broad waveband, and evolved quickly into a monochromatic oscillation with a period of about 5 minutes. The phase difference between the density and velocity perturbations was initially zero but rapidly became $\pi / 4$ (Figure 4(c)), which means a standing slow wave was formed within the resonator (Wang 2011; Yuan et al. 2015b). At the coronal height, we extracted time series at $z=10 \mathrm{Mm}(T \sim 0.8 \mathrm{MK})$. The signal was quasi-periodic (Figure 4(f)); and density and velocity oscillated almost in phase (Figure 4(d)), meaning that a propagating slow wave was observed at coronal heights (also see Wang et al. 2009a, 2009b; Yuan \& Nakariakov 2012; Fang et al. 2015). The average speed of propagation was about $100 \mathrm{~km} \mathrm{~s}^{-1}$. Owing to the lack of persistent energy supply, the slow wave decayed within a few cycles.

Our result is consistent with the theory of dispersive evolution of an impulsive disturbance in a stratified atmosphere (Chae \& Goode 2015; Kwak et al. 2016). However, we find a longer period (or lower resonance frequency) than Botha et al. (2011); this is because we used a less wild temperature profile, and thus a relatively smaller density gradient. It also implies that the resonance frequency is a good probe of the resonator's thermal structure, which may evolve with the age of a sunspot or other solar structures (Zhugzhda et al. 1983; Zhugzhda 2008). We note that periodic drivers are applied in the simulations of Heggland et al. (2007); the effect of the resonator is barely seen. This may be owing to the fact that they aim at simulating shock waves with large amplitudes and thus render the trapped energy less prominent. However, irregularities in the shock fronts could be seen in Figure 11 of Heggland et al. (2007), which may be a signature of the trapped energy. 


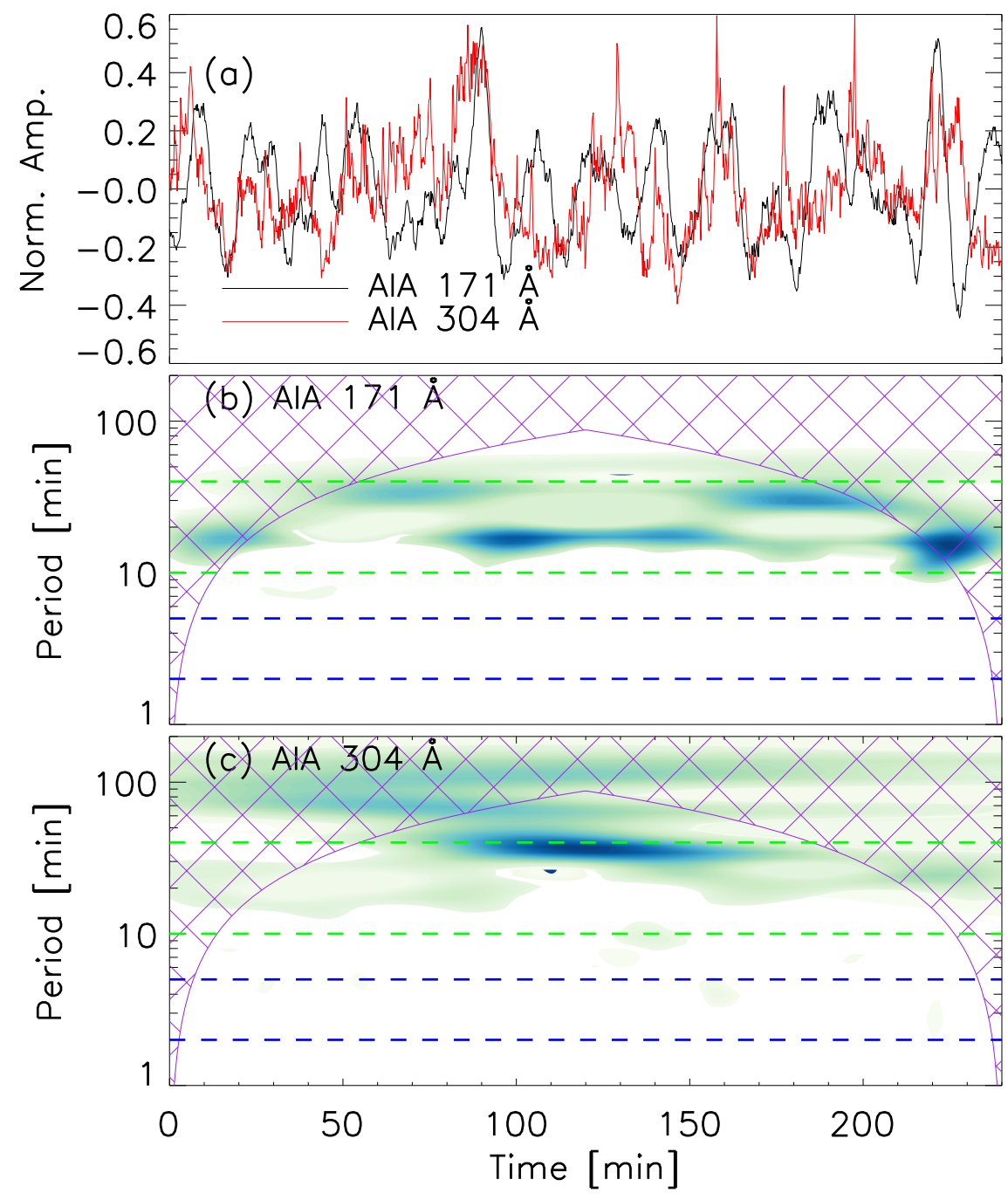

Figure 11. Same analysis as in Figure 8, but for the time series extracted at a plume as labeled in Figure 10. Two green dashed lines label the quasi-periodic longperiod oscillations at 10-30 minutes, whereas two blue dashed lines enclose oscillations in the band at 3 minutes. The cone-of-influence is cross-hatched; within it the spectrum should be considered as unreliable.

\subsection{Perturbation by Stochastic Transients}

After the single-pulse experiment, we applied the model of stochastic transients (Equation (1)); time series and parameters are illustrated in Figure 1. Figure 5 presents time-distance plots of density and velocity, and the time series and relevant wavelet analysis. Shock waves are formed at a height where density drops dramatically (Figure 5(a)); the amplitude could be as large as $60 \%$ of the background density (Figure 5(c)). At $z=1.8 \mathrm{Mm}$, the phase between density and velocity perturbations shows mixed features, while at $z=10 \mathrm{Mm}$, it is almost zero, indicating that propagating slow waves are found at the coronal height. The wavelet spectrum reveals that at $z=1.8$, long-period oscillations (10-30 minutes) and the resonance period ( $\sim 5$ minutes) coexist within the acoustic resonator (Figure 5(e)), while only the resonance period is allowed to leak from the chromosphere (Figure 5(f)). This is consistent with Snow et al. (2015), who demonstrated that even if long-period oscillations are implemented in the driver, their power would be $2-3$ orders of magnitude smaller than the resonance period. Long-period oscillations are indeed observed at ARs and other coronal structures (Marsh et al. 2009; Wang et al. 2009b; Yuan et al. 2011); this effect is ascribed to modification of the cut-off period of the slow wave by inclined magnetic field; see Bel \& Leroy (1977), McIntosh \& Jefferies (2006), Jess et al. (2013), Yuan et al. (2014b), and Afanasyev \& Nakariakov (2015).

We also notice that the resonance within the acoustic cavity and the leakage to the corona are not persistent, but form a series of wave trains. This is a novel feature that no other study has been able to simulate, and it is consistent with the fact that propagating disturbances at AR loops are detected in the form of wave trains (De Moortel 2009; De Moortel \& Nakariakov 2012). Moreover, the mixture of both long-period and short-period oscillations in the acoustic resonator is remarkably similar to the sunspot oscillations observed at chromospheric height using the Nobeyama RadioHeliograph (Chorley et al. 2010, 2011).

Long-period oscillations are also detected in coronal plumes (Ofman et al. 1999; Gupta et al. 2010; Krishna Prasad et al. 2011; Jiao et al. 2015). Low in the corona, the magnetic field pressure dominates over gas pressure, and a plume could simply be assumed to be isothermal (Del Zanna et al. 1998). In this study, we do not use an isothermal model, but simply apply the driver at $z=2.3 \mathrm{Mm}$, and demonstrate that altering the 


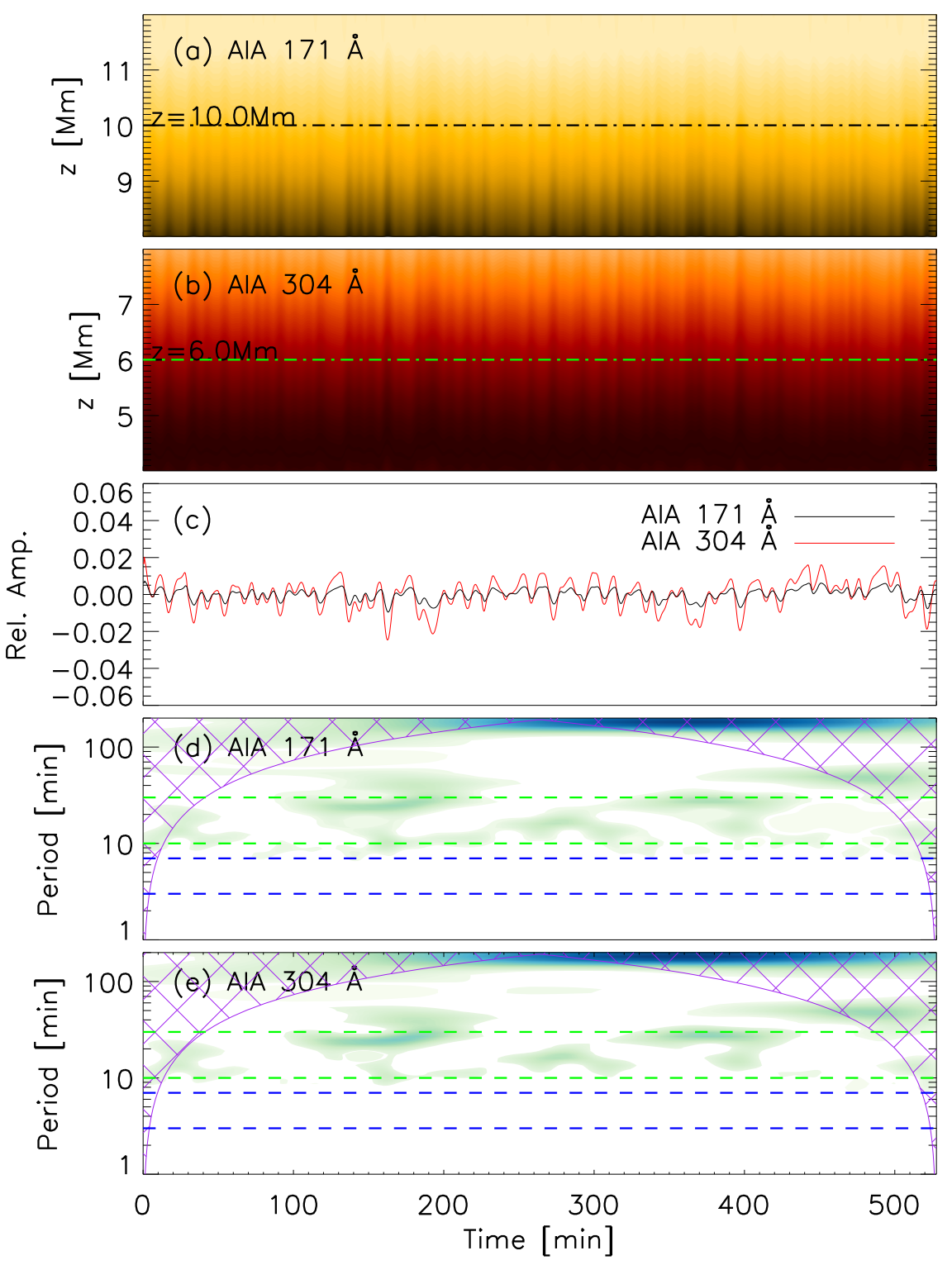

Figure 12. Same as Figure 9, but for the simulation with a driver applied at $z=2.3 \mathrm{Mm}$. The cone-of-influence is cross-hatched; within it the spectrum should be considered as unreliable. Two green dashed lines label the quasi-periodic long-period oscillations introduced by the stochastic driver as illustrated in Figure 1, whereas two blue dashed lines enclose the range of resonance period, as shown in Figure 4.

thermal structure of the solar atmosphere could also lead to the leakage of long-period oscillations.

Figure 6 presents the result if we apply the driver at $z=2.3 \mathrm{Mm}(T=0.1 \mathrm{MK})$; this scenario skips the chromospheric cavity. In contrast to when the driver is launched deeper, the density and velocity perturbations do not form shock waves, but their amplitude remains small (Figures 6(c) and (d)). The long-period wave trains manage to propagate to coronal heights.

\section{OBSERVATIONS IN THE SOLAR ATMOSPHERE}

\subsection{Oscillations in the Loops of Sunspots and Active Regions}

In the solar atmosphere, the thermal structure of a sunspot and the associated AR resembles the empirical atmospheric model used (see Section 3.1). Therefore, we selected a $5 \mathrm{hr}$ AIA observation on AR 11504 (Figure 7). The start time is 16:00:00 UT on 2012 June 14. We used the AIA 304 and $171 \AA$ channels, which have nominal response temperatures at $85,000 \mathrm{~K}$ (He II) and 850,000 K (Fe IX), respectively (Boerner et al. 2012). This AR has already been studied intensively in $\mathrm{Su}$ et al. (2013). A coronal loop anchoring at the trailing spot of AR 11504 was tracked (Figure 7). We extracted time series of the emission intensity in AIA 304 and $171 \AA$, removed the trend of a 30 minute running average, and normalized the amplitude to \pm 0.5 . Figure 8 plots the time series and the relevant wavelet analysis. We cannot guarantee that two time series were taken at two different heights of the same loop, but the oscillations at diffuse coronal structures have a correlation area about $10 \mathrm{Mm}$ long and a few $\mathrm{Mm}$ wide (De Moortel et al. 2002b), within which the wavelet spectrum is only slightly altered. So the ambiguity due to the projection effect will not affect our conclusions.

We could see that in both AIA 304 and $171 \AA$ channels, one could detect prominent oscillations at 3 minutes in the form of wave trains (Figure 8), which has been reported in a number of 
studies (e.g., De Moortel et al. 2002a; King et al. 2003; Yuan et al. 2014b). Meanwhile, we also detect significant long-period oscillations at 10-30 minutes at both heights. The leakage of long-period oscillations was studied previously, e.g., Yuan et al. (2011), Marsh et al. (2009), and Wang et al. (2009b). The mechanism for the leakage is not fully understood: the inclined magnetic field could lower the cut-off frequency (De Pontieu et al. 2005; McIntosh \& Jefferies 2006; Yuan et al. 2014b), evanescent slow waves with long penetration depths could regain a propagating wave feature at coronal heights (Fleck \& Schmitz 1991; Yuan et al. 2011), or long-period oscillations are somehow generated at coronal heights.

To compare with the observational data, we synthesized the AIA 304 and $171 \AA$ emission intensities (details of forward modeling are available in Fang et al. 2015; Yuan et al. 2015b; Van Doorsselaere et al. 2016), by using the CHIANTI atomic emission database (Dere et al. 1997; Landi et al. 2013). Figure 9 presents the time-distance plots, the time series extracted at $z=6 \mathrm{Mm}(304 \AA)$ and $10 \mathrm{Mm}(171 \AA)$ and the associated wavelet analysis. The time series for the synthetic AIA 304 and $171 \AA$ emission intensities exhibit oscillations only at the resonance period ( $\sim 5$ minutes), while long-period oscillations are occasionally detected at a low confidence level. In our simulation, the magnetic field line is vertical; the magnetoacoustic cut-off frequency is not modified at all. In an $\mathrm{AR}$, magnetic fields are bent to connect to the opposite polarity, so significant long-period oscillations are more likely to be detected (Yuan et al. 2011, 2014b).

\subsection{Propagating Disturbances in Polar Plumes}

To investigate the oscillations in polar plumes, we select a set of $4 \mathrm{hr}$ AIA observations on a coronal hole, starting from 21:50 UT on 2010 August 5. A few polar plumes are identifiable low in the corona in the AIA $171 \AA$ channel, and their bases could be well traced in the AIA $304 \AA$ channel (Figure 10). The plume structure enclosed in the rectangle is chosen for further analysis. We extracted the average emission intensity within the small boxes in the 304 and $171 \AA$ FOVs, respectively. We used the original light curves of the $304 \AA$ bandpass, while for the $171 \AA$ bandpass, we removed a trend of the 30 minutes moving average and smoothed the time series. The amplitudes are normalized to $[ \pm 0.5]$. Long-period oscillations at 10-30 minutes are apparent in the light curve and wavelet spectrum of 304 and $171 \AA$ (Figure 11).

Similar procedures are implemented for the simulation with a driver launched at $z=2.3 \mathrm{Mm}$. Figure 12 illustrates the result of forward modeling to compare with oscillations in polar plumes. Indeed, the long-period oscillations propagate to coronal height and are detectable in the UV/EUV bandpasses.

In the simulation, we use only the timescale of spicular activities that are found to be highly correlated with quasiperiodic propagating disturbances in polar plumes (Jiao et al. 2015; Samanta et al. 2015). However, as Krishna Prasad et al. (2012) shows, multiple periods coexist in the propagating disturbances; therefore other timescales are needed to fully address this issue.

\section{CONCLUSIONS}

In this study, we propose that stochastic, finite-lifetime transients could generate quasi-periodic processes in the solar atmosphere, and we suggest a mathematical model of stochastic transients in the form of Gaussian profiles. Then we use the typical timescales of spicular activities and simulate the propagation of stochastic transients in an empirical model of the atmosphere. The existence of a chromospheric resonator filters out the long-period oscillations, and only the resonance period is able to propagate to the upper atmosphere. Observations with SDO/AIA 304 and $171 \AA$ bandpasses, which are sensitive to the chromospheric and coronal heights, produce consistent results. We also investigate the case in which the thermal structure is changed into a smoother form and the acoustic resonator does not exist. Such a scenario is applicable to polar plumes. Indeed, both simulations and observations clearly measure significant long-period oscillations.

Our model is applicable to many field in geophysics, solar physics, stellar physics, and astrophysics. The timescale could be scaled to any range of interest; we only provide a possible application.

The research was supported by the National Basic Research Program of China under grant 11373040 and the Open Research Program KLSA201504 of Key Laboratory of Solar Activity of National Astronomical Observatories of China.

\section{REFERENCES}

Afanasyev, A. N., \& Nakariakov, V. M. 2015, A\&A, 582, A57

Anfinogentov, S., Nisticò, G., \& Nakariakov, V. M. 2013, A\&A, 560, A107

Anfinogentov, S. A., Nakariakov, V. M., \& Nisticò, G. 2015, A\&A, 583, A136

Avrett, E. H., \& Loeser, R. 2008, ApJS, 175, 229

Banerjee, D., Gupta, G. R., \& Teriaca, L. 2011, SSRv, 158, 267

Beckers, J. M. 1968, SoPh, 3, 367

Bel, N., \& Leroy, B. 1977, A\&A, 55, 239

Boerner, P., Edwards, C., Lemen, J., et al. 2012, SoPh, 275, 41

Botha, G. J. J., Arber, T. D., Nakariakov, V. M., \& Zhugzhda, Y. D. 2011, ApJ, 728, 84

Cargill, P. J., Warren, H. P., \& Bradshaw, S. J. 2015, RSPTA, 373, 20140260

Chae, J., \& Goode, P. R. 2015, ApJ, 808, 118

Chorley, N., Foullon, C., Hnat, B., Nakariakov, V. M., \& Shibasaki, K. 2011, A\&A, 529, A123

Chorley, N., Hnat, B., Nakariakov, V. M., Inglis, A. R., \& Bakunina, I. A. 2010, A\&A, 513, A27

De Moortel, I. 2009, SSRv, 149, 65

De Moortel, I., Ireland, J., Hood, A. W., \& Walsh, R. W. 2002a, A\&A, 387, L13

De Moortel, I., Ireland, J., Walsh, R. W., \& Hood, A. W. 2002b, SoPh, 209, 61 De Moortel, I., \& Nakariakov, V. M. 2012, RSPTA, 370, 3193

De Pontieu, B., Erdélyi, R., \& De Moortel, I. 2005, ApJL, 624, L61

De Pontieu, B., Hansteen, V. H., Rouppe van der Voort, L., van Noort, M., \& Carlsson, M. 2007a, ApJ, 655, 624

De Pontieu, B., \& McIntosh, S. W. 2010, ApJ, 722, 1013

De Pontieu, B., McIntosh, S., Hansteen, V. H., et al. 2007b, PASJ, 59, S655

Del Zanna, G. 2008, A\&A, 481, L49

Del Zanna, L., von Steiger, R., \& Velli, M. 1998, SSRv, 85, 349

Dere, K. P., Landi, E., Mason, H. E., Monsignori Fossi, B. C., \& Young, P. R. 1997, A\&AS, 125, 149

Doschek, G. A., Warren, H. P., Mariska, J. T., et al. 2008, ApJ, 686, 1362

Fang, X., Yuan, D., Van Doorsselaere, T., Keppens, R., \& Xia, C. 2015, ApJ, 813,33

Fleck, B., \& Schmitz, F. 1991, A\&A, 250, 235

Gupta, G. R., Banerjee, D., Teriaca, L., Imada, S., \& Solanki, S. 2010, ApJ, 718,11

Hannah, I. G., Hudson, H. S., Battaglia, M., et al. 2011, SSRv, 159, 263

Hansteen, V. H., De Pontieu, B., Rouppe van der Voort, L., van Noort, M., \& Carlsson, M. 2006, ApJL, 647, L73

Hara, H., Watanabe, T., Harra, L. K., et al. 2008, ApJL, 678, L67

Harten, A., Lax, P. D., \& van Leer, B. 1983, SIAMR, 25, 35

Heggland, L., De Pontieu, B., \& Hansteen, V. H. 2007, ApJ, 666, 1277

Jess, D. B., Reznikova, V. E., Van Doorsselaere, T., Keys, P. H., \& Mackay, D. H. 2013, ApJ, 779, 168

Jiao, F., Xia, L., Li, B., et al. 2015, ApJL, 809, L17 
Keppens, R., Meliani, Z., van Marle, A. J., et al. 2012, JCoPh, 231, 718

Kiddie, G., De Moortel, I., Del Zanna, G., McIntosh, S. W., \& Whittaker, I. 2012, SoPh, 279, 427

King, D. B., Nakariakov, V. M., Deluca, E. E., Golub, L., \& McClements, K. G. 2003, A\&A, 404, L1

Klimchuk, J. A. 2015, RSPTA, 373, 20140256

Koren, B. 1993, A Robust Upwind Discretization Method for Advection, Diffusion and Source Terms (Amsterdam: Centrum voor Wiskunde en Informatica)

Krishna Prasad, S., Banerjee, D., \& Gupta, G. R. 2011, A\&A, 528, L4

Krishna Prasad, S., Banerjee, D., Van Doorsselaere, T., \& Singh, J. 2012, A\&A, 546, A50

Kwak, H., Chae, J., Song, D., et al. 2016, ApJL, 821, L3

Landi, E., Young, P. R., Dere, K. P., Del Zanna, G., \& Mason, H. E. 2013, ApJ, 763, 86

Liu, W., Ofman, L., Nitta, N. V., et al. 2012, ApJ, 753, 52

Liu, W., Title, A. M., Zhao, J., et al. 2011, ApJL, 736, L13

Maltby, P., Avrett, E. H., Carlsson, M., et al. 1986, ApJ, 306, 284

Marsh, M. S., Walsh, R. W., \& Plunkett, S. 2009, ApJ, 697, 1674

McIntosh, S. W., \& De Pontieu, B. 2009, ApJ, 707, 524

McIntosh, S. W., Innes, D. E., de Pontieu, B., \& Leamon, R. J. 2010, A\&A, 510, L2

McIntosh, S. W., \& Jefferies, S. M. 2006, ApJL, 647, L77

Nakariakov, V. M., Verwichte, E., Berghmans, D., \& Robbrecht, E. 2000, A\&A, 362, 1151

Nisticò, G., Nakariakov, V. M., \& Verwichte, E. 2013, A\&A, 552, A57

Nordlund, A., Stein, R. F., \& Asplund, M. 2009, LRSP, 6, 2

Ofman, L., Nakariakov, V. M., \& DeForest, C. E. 1999, ApJ, 514, 441

Ofman, L., Wang, T. J., \& Davila, J. M. 2012, ApJ, 754, 111

Parker, E. N. 1988, ApJ, 330, 474

Pascoe, D. J., Nakariakov, V. M., \& Kupriyanova, E. G. 2013, A\&A, 560, A97

Porter, J. G., Toomre, J., \& Gebbie, K. B. 1984, ApJ, 283, 879

Porth, O., Xia, C., Hendrix, T., Moschou, S. P., \& Keppens, R. 2014, ApJS, 214, 4

Rieutord, M., \& Rincon, F. 2010, LRSP, 7, 2

Roberts, B. 2006, RSPTA, 364, 447

Rouppe van der Voort, L., Leenaarts, J., de Pontieu, B., Carlsson, M., \& Vissers, G. 2009, ApJ, 705, 272
Samanta, T., Pant, V., \& Banerjee, D. 2015, ApJL, 815, L16

Shen, Y., \& Liu, Y. 2012, ApJ, 753, 53

Shen, Y.-D., Liu, Y., Su, J.-T., et al. 2013, SoPh, 288, 585

Skogsrud, H., Rouppe van der Voort, L., \& De Pontieu, B. 2016, ApJ, 817,124

Snow, B., Botha, G. J. J., \& Régnier, S. 2015, A\&A, 580, A107

Stein, R. F. 2012, LRSP, 9, 4

Sterling, A. C. 2000, SoPh, 196, 79

Sterling, A. C., Moore, R. L., \& DeForest, C. E. 2010, ApJL, 714, L1

Su, J. T., Ji, K. F., Banerjee, D., et al. 2016a, ApJ, 816, 30

Su, J. T., Ji, K. F., Cao, W., et al. 2016b, ApJ, 817, 117

Su, J. T., Liu, Y., Liu, S., et al. 2013, ApJ, 762, 42

Tian, H., DeLuca, E., Reeves, K. K., et al. 2014, ApJ, 786, 137

Tian, H., McIntosh, S. W., \& De Pontieu, B. 2011, ApJL, 727, L37

Torrence, C., \& Compo, G. P. 1998, BAMS, 79, 61

Uritsky, V. M., Davila, J. M., Viall, N. M., \& Ofman, L. 2013, ApJ, 778, 26

Van Doorsselaere, T., Antolin, P., Yuan, D., Reznikova, V., \& Magyar, N. 2016, FrASS, 3, 4

Verwichte, E., Marsh, M., Foullon, C., et al. 2010, ApJL, 724, L194

Wang, T. 2011, SSRv, 158, 397

Wang, T., Ofman, L., \& Davila, J. M. 2013, ApJL, 775, L23

Wang, T. J., Ofman, L., \& Davila, J. M. 2009a, ApJ, 696, 1448

Wang, T. J., Ofman, L., Davila, J. M., \& Mariska, J. T. 2009b, A\&A, 503, L25

Yang, L., Zhang, L., He, J., et al. 2015, ApJ, 800, 111

Yuan, D., \& Nakariakov, V. M. 2012, A\&A, 543, A9

Yuan, D., Nakariakov, V. M., Chorley, N., \& Foullon, C. 2011, A\&A, 533, A116

Yuan, D., Nakariakov, V. M., Huang, Z., et al. 2014a, ApJ, 792, 41

Yuan, D., Pascoe, D. J., Nakariakov, V. M., Li, B., \& Keppens, R. 2015a, ApJ, 799, 221

Yuan, D., Shen, Y., Liu, Y., et al. 2013, A\&A, 554, A144

Yuan, D., Sych, R., Reznikova, V. E., \& Nakariakov, V. M. 2014b, A\&A, 561, A19

Yuan, D., Van Doorsselaere, T., Banerjee, D., \& Antolin, P. 2015b, ApJ, 807, 98

Zhang, Y. Z., Shibata, K., Wang, J. X., et al. 2012, ApJ, 750, 16

Zhugzhda, I. D., Locans, V., \& Staude, J. 1983, SoPh, 82, 369

Zhugzhda, Y. D. 2008, SoPh, 251, 501 